\title{
Sustainability of Biologic Therapy is Less in Ulcerative Colitis than Crohn's Disease Patients, Independent of Prior Biologic Experience
}

\author{
Jayne Doherty ${ }^{*}$, Maire Buckley ${ }^{1,3}$, Garret Cullen ${ }^{1,2}$, Denise Keegan ${ }^{1}$, Kathryn Byrne', Gareth \\ Horgan $^{1,4}$, Hugh Mulcahy ${ }^{1,2}$, Juliette Sheridan ${ }^{1,2}$ and Glen A Doherty ${ }^{1,2}$ \\ ${ }^{1}$ Centre for Colorectal Disease, St Vincent's University Hospital \& School of Medicine, University College Dublin, Ireland \\ ${ }^{2}$ St Vincent's Private Hospital, Elm Park, Dublin 4, Ireland \\ ${ }^{3}$ St Michael's Hospital, Dunlaoighre, Dublin, Ireland \\ ${ }^{4}$ St Columcille's Hospital, Loughlinstown, Dublin, Ireland
}

*Corresponding author: Jayne Doherty, Centre for Colorectal Disease, St Vincent's University Hospital \& School of Medicine, University College Dublin, Elm Park, Dublin 4, Ireland

\section{ARTICLE INFO}

Received: 幽 July 23, 2021

Published: 幽 August 06, 2021

Citation: Jayne Doherty, Maire Buckley, Garret Cullen, Denise Keegan, Kathryn Byrne, et al., Sustainability of Biologic Therapy is Less in Ulcerative Colitis than Crohn's Disease Patients, Independent of Prior Biologic Experience. Biomed J Sci \& Tech Res 37(5)-2021. BJSTR. MS.ID.006071.

Keywords: Inflammatory Bowel Disease; Biologic Therapy; Loss of Response

Abbreviations: IBD: Inflammatory Bowel Disease; UC: Ulcerative Colitis; CD: Crohn's Disease; TNF: Anti-Tumour Necrosis Factor; JAK: Janus Kinase; IL: Anti-Interleukins; CI: Confidence Interval; IQR: Interquartile Range; IFX: Infliximab; ADA: Adalimumab; GLM: Golimumab; UST: Ustekinumab; HR: Hazard Ratio; US: United States

\section{ABSTRACT}

Background: Treatment of Inflammatory Bowel Disease (IBD) with biologics is effective but loss of response to treatment limits long-term treatment success. Few studies have examined what determines sustainability of biologic treatment.

Objective: The aim of our study was to determine factors associated with sustainability of biologic therapy in IBD.

Methods: We performed a retrospective study of 4,200 patients in a single healthcare network. Primary outcome was time to biologic treatment discontinuation (due to inadequate treatment response or adverse effects).

Results: A total of 948 independent biologic treatment episodes were identified in 712 patients. Mean follow-up was 2.3years (range $0.0028-5.00$ ).

- Group 1: 701 biologic-naïve treatment episodes were included. 329 patients (47\%) were treated with infliximab, 337(48\%) with adalimumab and 32(5\%) with golimumab. $250(36 \%)$ had UC. Mean time to discontinuation in UC was 2.9 years compared to 3.6 years in $C D(p=<0.001)$.

- Group 2: 247 treatment episodes of biologic-experienced patients were included. $83(33 \%)$ were treated with infliximab, 57(23\%) with adalimumab, $40(16 \%)$ with vedolizumab, 26(11\%) with golimumab and 41(16.6\%) with ustekinumab. 99(40\%) had UC. Mean time to discontinuation in UC was 2.3 years compared to 3.1 years in CD ( $\mathrm{p}=$ 0.03).

Conclusion: Our real-world data indicates sustainability of biologic treatment is less in UC than in CD patients and is not strongly determined by prior biologic exposure. These findings suggest the need for better understanding of the differing mechanisms for loss of biologic response which will assist in sequencing of therapies in the future.

\section{Key Summary}

Loss of response to biologic agents is common in clinical practice. To date factors impacting response to anti-TNF agents include gender, disease duration and the presence of fistulating disease in Crohn's disease. The impact of subtype on IBD on response to biologic agents has not previously been investigated. In our paper we found that in biologic agents were more durable in Crohn's disease compared to ulcerative colitis. In both biologic naïve and biologic experienced patients time to discontinuation of biologic therapy was significantly shorter in patients with ulcerative colitis compared with Crohn's disease. 


\section{Introduction}

Inflammatory bowel disease (IBD) is a chronic inflammatory condition that predominantly affects the bowel and includes both Ulcerative Colitis (UC) and Crohn's Disease (CD). The burden of IBD is rising globally with a marked increase in the prevalence of IBD worldwide over the last 30 years [1]. The therapeutic goal in the treatment of IBD has evolved from focusing on symptomatic relief to aiming to achieve intestinal mucosal healing. Emerging longitudinal data suggests that individuals who obtain mucosal healing, which is observed more frequently with biologic than conventional therapies, have a superior long-term prognosis with lower risk of surgery and hospitalization [2]. While there has been an expansion in the number of medications available for treating IBD including anti-tumour necrosis factor (TNF) agents, selective anti-integrins, anti-interleukins (IL) and most recently small molecule inhibitors such as the janus kinase (JAK) inhibitors, there still remains a large unmet clinical need for further therapies. Studies have shown between one third to half of patients commenced on anti-TNFs do not maintain response and require a switch to a second biologic [3]. Reasons for discontinuation vary but are often secondary to nonsustained response to a particular agent (primary non-response and secondary loss of response) or development of intolerance to a particular biologic. Chen et al looked at over 8000 patients with IBD on biologics and found after 1 year less than $50 \%$ of patients were still on this biologic agent [4].

With the evolution of medical management of IBD and new treatment goals there is a need to identify factors that predict response to biologics. To date age at diagnosis, disease duration, disease severity, and elevated inflammatory markers are all factors that have been identified to influence response to biologics [5]. However, few studies have specifically looked at real world sustainability of response to biologics, factors which might determine this and whether there is a difference between UC and CD. Given the distinct biological differences between UC and CD we aimed to look at the difference in sustainability between biologics in biologic-naïve and biologic-experienced patients depending on whether they had a diagnosis of UC or CD.

\section{Material and Methods}

\section{Participants}

A prospectively maintained electronic database of 4200 patients with IBD attending four hospitals in a single healthcare network was interrogated from January 2008 to March 2018. All patients with an established diagnosis of CD or UC currently and previously on biologic therapy were included in this analysis.

\section{Study Design}

Basic demographic information was collected including age, gender, disease subtype, age at diagnosis, type of biologic therapy used and concurrent immunomodulator therapy. Date patients commenced on a particular biologic was collected along with the date of discontinuation of biologic therapy (or last follow-up date if patients were still on this agent) due to inadequate treatment response or adverse effects. Where treatment was discontinued for other reasons (patient choice, sustained remission, or nonadherence) these treatment episodes were excluded. Whether a patient was biologic-naïve or had prior treatment with a different biologic agent (biologic-experienced) was also recorded for analysis. Ethical approval for this study was granted by the Ethics Committee at St Vincent's University Hospital.

\section{Outcomes}

The primary outcome of interest was time to discontinuation of biologics in biologic-naïve and biologic-experienced patients' dependent on whether patients were diagnosed of UC (UC and IBD-U) or CD. Secondary outcomes of interest included the identification of disease characteristics that impact discontinuation of biologics including immunomodulator co-therapy, age at diagnosis, disease duration, gender and disease location for both UC and CD.

\section{Statistical Methods}

Descriptive statistics were provided to summarize demographic characteristics using mean [95\% confidence interval (CI)] or median [interquartile range (IQR)] for continuous variables, and number and percentage for categorical variables. Cumulative survival rates were estimated using the Kaplan-Meier method and mean and median survival time used. The log-rank test was used to compare the survival curves across the subgroups. A multivariate (adjusted analysis) Cox proportional hazards model was utilized to identify risk factors relating to survival. A two-sided P-value $\leq$ 0.05 was considered statistically significant. All data analyses were performed using SPSS 25.0.

\section{Results}

\section{Baseline Characteristics}

A total of 1081 treatment events with biologic therapy occurred at our centre at the time of this study. 47 events were excluded due to lack of data and 86 were excluded due to discontinuation of a biologic agent for reasons other than loss of response. In total 948 independent treatment events with biologic therapy were included in this study in 712 patients. In total 516 (54.4\%) patients were male. Median age at diagnosis was 33.8 years (IQR 24.6 - 44.8). $349(36.8 \%)$ patients had a diagnosis of UC. 353 (37.2\%) patients in total discontinued their biologic agents. 312 (32.9\%) patients were on co-therapy with immunomodulators. Mean follow-up was 2.3 years (IQR 0.64-3.2) (Table 1). Patients were sub-divided depending on whether they were biologicnaïve (Group1) or biologic experienced (Group 2) (Figure 1). 


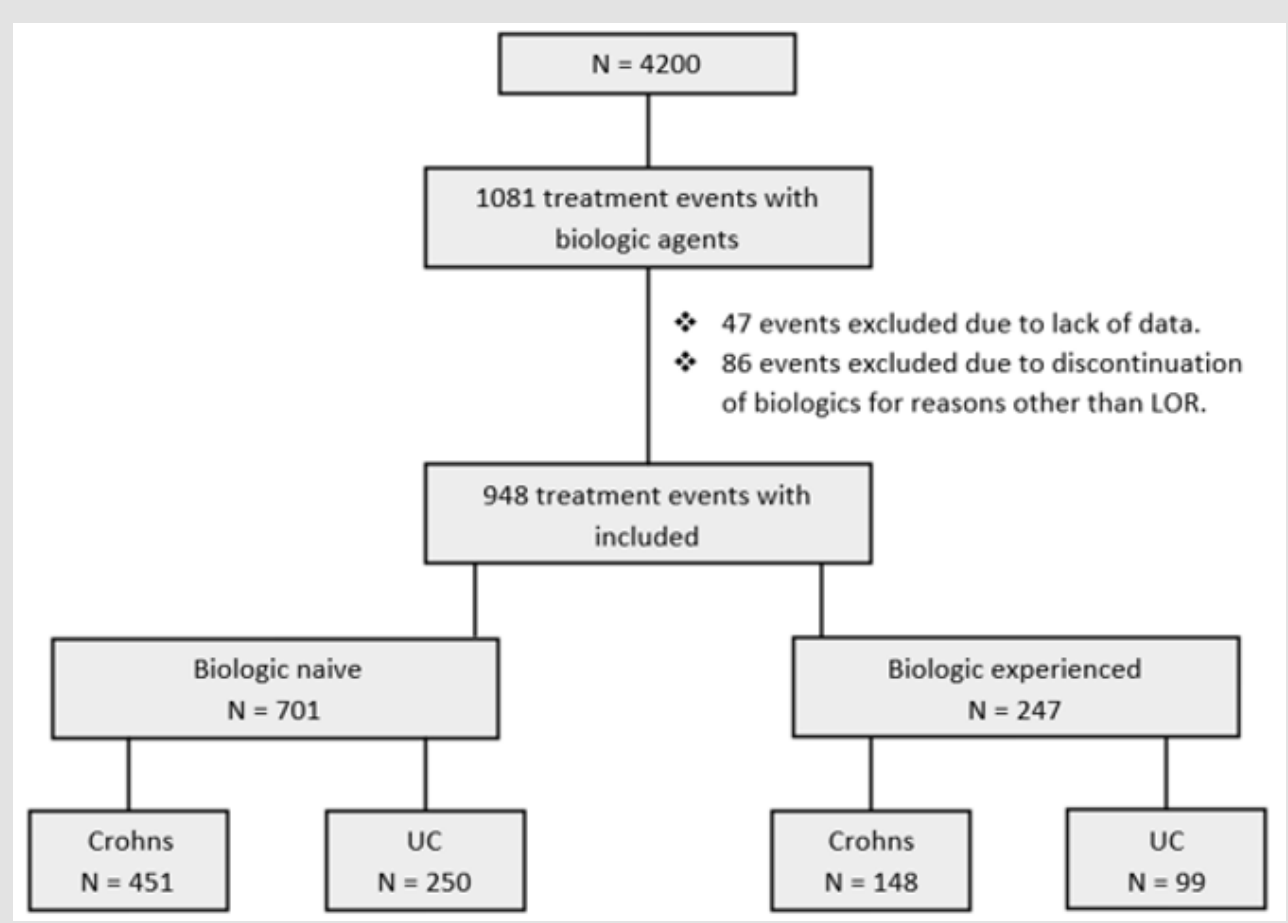

Figure 1: Flow diagram of patient selection.

Table 1: Baseline characteristic.

\begin{tabular}{|c|c|c|c|c|}
\hline & Total (n = 948) & Biologic-naïve (n= 701) & Biologic-experienced (n= 247) & P-value \\
\hline Male & $516(54 \%)$ & $387(55 \%)$ & $129(52 \%)$ & 0.44 \\
\hline Median age at diagnosis & 33.75 & 33.25 & 34.9 & 0.28 \\
\hline UC & $349(37 \%)$ & $250(36 \%)$ & $99(40 \%)$ & 0.22 \\
\hline Discontinued biologics & $353(37 \%)$ & $256(37 \%)$ & $97(39 \%)$ & 0.4 \\
\hline Immunomodulators & $312(33 \%)$ & $215(31 \%)$ & $97(39 \%)$ & 0.002 \\
\hline
\end{tabular}

\section{Sustainability of Biologics Between UC and CD}

- $\quad$ Group 1: 701 (74 \%) patients were biologic-naïve. 387 (55.2\%) were male. Median age at diagnosis was 33.3 years (IQR 23.7 - 44.5). 250 (35.7 \%) had a diagnosis of UC and 451 (64.3\%) had CD. Median disease duration was 7.5 years (IQR 3.1 - 13.9). 256 (36.5 \%) patients discontinued their biologics. 215 (30.7\%) patients were on co-therapy with immunomodulators (Table 1). 329 (46.9\%) patients received infliximab (IFX), 337 (48.1\%) adalimumab (ADA), 32 (4.6\%) golimumab (GLM) and 3 (0.4\%) received ustekinumab (UST). Mean time to discontinuation of biologics in the UC cohort was 2.9 years (95\% CI 2.6 - 3.22 years) which was significantly shorter than in the CD cohort which was 3.6 years (95\% CI $3.4-3.8$ years) (p-value $=<0.001$ ) (Table $2 \&$ Figure
2).

- $\quad$ Group 2: 247 (26.1\%) patients were biologic experienced. 129 (52.2 \%) were male. Median age at diagnosis was 34.9 years (IQR 27.8 - 45.0). 99 (40.1\%) had a diagnosis of UC and 148 (59.9 $\%$ ) had CD. Median disease duration was 10.5 years (IQR 5.2 15.9). 97 (39.3\%) patients discontinued their biologics. 97 (39.3 $\%)$ patients were on co-therapy with immunomodulators (Table 1). 83 (33.6\%) patients received IFX, 57 (23.1\%) ADA, 40 (16.2\%) vedolizumab, 26 (10.5\%) GLM and 41 (16.6\%) received UST. Mean time to discontinuation of biologics in the CD cohort was 3.1 years (95\% CI 2.7 - 3.5 years) compared with 2.3 years (95\% CI $1.9-2.8$ years) in the UC cohort ( $\mathrm{p}$-value $=0.030$ ) (Table $3 \&$ Figure 3 ). 


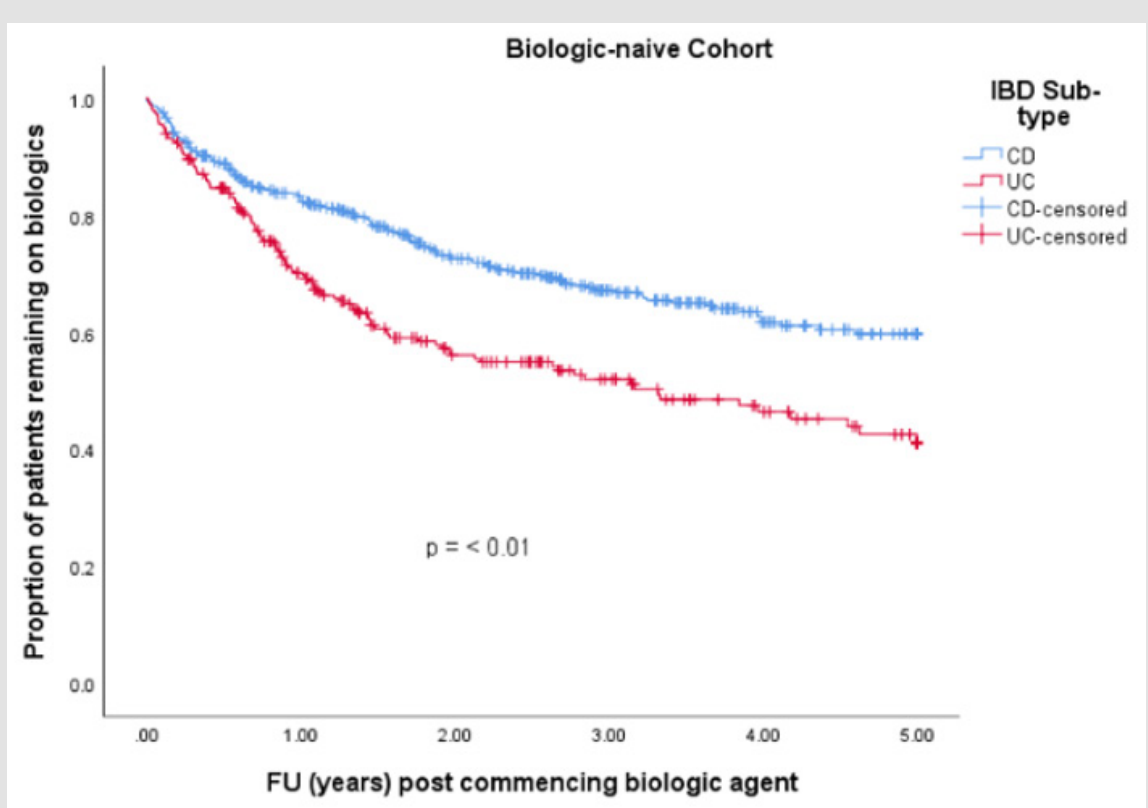

Figure 2: Time to discontinuation of biologic agents in our biologic-naïve cohort.

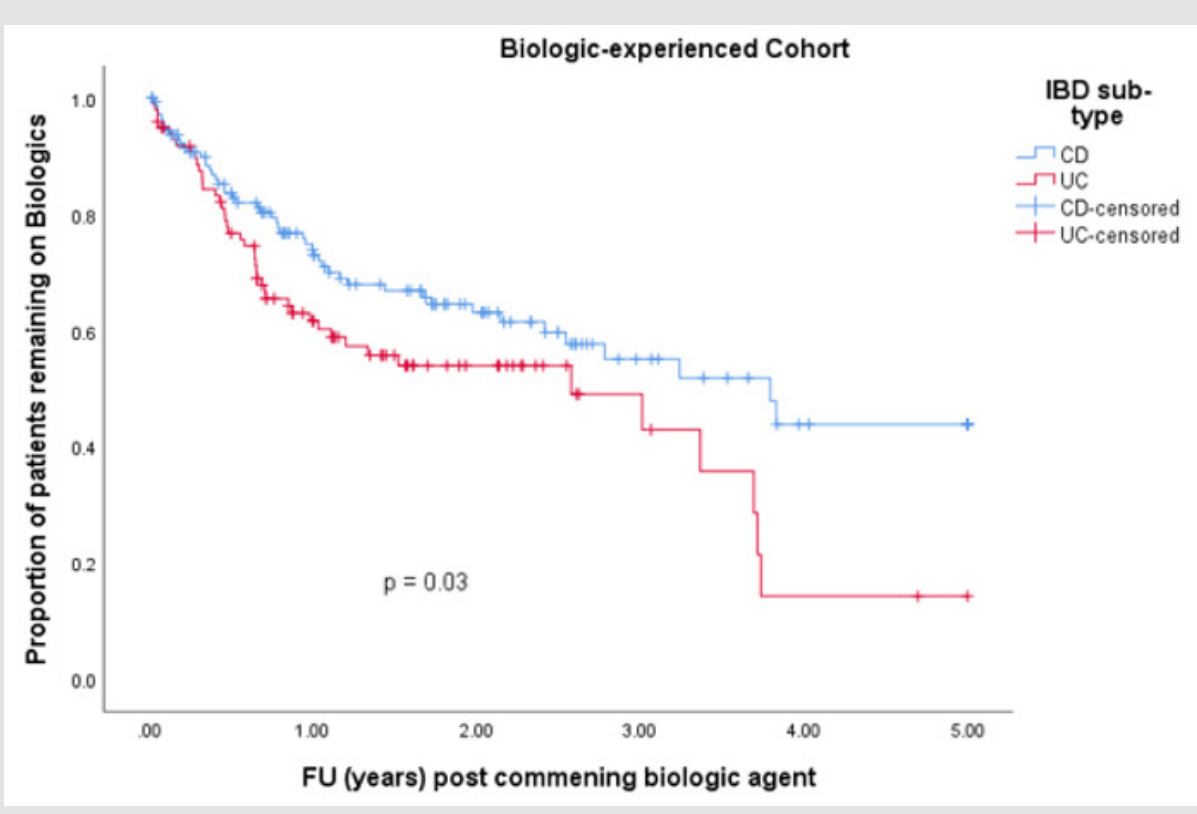

Figure 3: Time to discontinuation of biologic agents in our biologic-experienced cohort.

Table 2: Survival analysis biologic-naïve cohort.

\begin{tabular}{|c|c|c|c|}
\hline \multirow{2}{*}{ Biologic naïve cohort } & \multirow{2}{*}{$\begin{array}{c}\text { Mean time to } \\
\text { discontinuation (years) }\end{array}$} & Lower & \multirow{2}{*}{ P value } \\
\cline { 3 - 4 } & 3.63 & 3.44 & 3.82 \\
\hline CD & 2.94 & 2.66 & 3.22 \\
\hline
\end{tabular}


Table 3: Survival analysis biologic-experienced cohort.

\begin{tabular}{|c|c|c|c|c|}
\hline \multirow{2}{*}{$\begin{array}{l}\text { Biologic experienced } \\
\text { cohort }\end{array}$} & \multirow{2}{*}{$\begin{array}{c}\text { Mean time to } \\
\text { discontinuation (years) }\end{array}$} & \multicolumn{2}{|c|}{ 95\% Confidence Interval } & \multirow{2}{*}{$P$ value } \\
\hline & & & Upper & \\
\hline $\mathrm{CD}$ & 3.1 & 2.7 & 3.5 & \multirow{2}{*}{0.03} \\
\hline UC & 2.3 & 1.9 & 2.8 & \\
\hline
\end{tabular}

\section{Factors Affecting Discontinuation of Biologics}

We looked at gender, age at diagnosis, co-therapy with immunomodulators, IBD subtype and disease duration as potential factors impacting biologic discontinuation.

- Group 1: Gender had no impact on discontinuation of biologic agents with a hazard ratio (HR) 0.8 (p-value 0.07, 95\% CI 0.6-1.0). Older age at diagnosis was associated with a slight increased risk of discontinuing a biologic agent with a HR 1.009 (p-value 0.002, 95\% CI 1.00-1.02). Shorter disease duration was protective against discontinuation of biologics with a HR of 0.97 (p-value <0.001, 95\% CI 0.95 - 0.98). Co-therapy with immunomodulators increased the risk of discontinuing biologic therapy in a biologic-naïve cohort with a HR 1.6 (p-value 0.001, $95 \%$ CI 1.2 - 2.1). UC as seen in our survival analysis increased your risk of discontinuing biologic therapy in our biologic naïve cohort with a HR 1.5 (p-value $0.003,95 \%$ CI 1.2 - 1.9) (Table 4).

- $\quad$ Group 2: Neither gender nor age at diagnosis showed any independent association with time to discontinuation of biologics in our biologic-experienced cohort. Shorter disease duration was again protective against discontinuation of biologics with a HR of 0.95 (p-value 0.002, 95\% CI 0.92-0.98). Co-therapy with immunomodulator therapy had no significant impact on discontinuation of biologics in our biologic-experienced cohort with a HR 0.9 (p-value $0.8,95 \%$ CI 0.9 - 1.5) and neither did a diagnosis of UC (p-value $0.2,95 \%$ CI 0.9 - 2.1) (Table 5).

Table 4: Factors affecting discontinuation of biologics in biologic naïve cohort.

\begin{tabular}{|c|c|c|c|}
\hline Biologic naïve & B Co-efficient & Hazard Ratio & 95\% Confidence Interval \\
\hline Gender & -0.2 & 0.8 & $0.6-1.0$ \\
\hline Age at diagnosis & 0.01 & 1.009 & $1.00-1.02$ \\
\hline Disease Duration & -0.03 & 0.97 & $0.95-0.98$ \\
\hline Immunomodulators & 0.46 & 1.6 & $1.2-2.1$ \\
\hline UC & 0.4 & 1.5 & 0.001 \\
\hline
\end{tabular}

Table 5: Factors affecting discontinuation of biologics in biologic experienced cohort.

\begin{tabular}{|c|c|c|c|c|}
\hline Biologic experienced & B Co-efficient & Hazard Ratio & 95\% Confidence Interval & P-value \\
\hline Gender & 0.2 & 1.3 & $0.8-1.9$ & 0.3 \\
\hline Age at diagnosis & -0.001 & 1 & $0.98-1.0$ & 0.88 \\
\hline Disease Duration & -0.05 & 0.95 & $0.92-0.98$ & 0.002 \\
\hline Immunomodulators & -0.6 & 0.9 & $0.9-1.5$ & 0.8 \\
\hline UC & 0.3 & 1.4 & $0.9-2.1$ & 0.2 \\
\hline
\end{tabular}

\section{Discussion}

Over the past two decades there has been significant advances in the medical management of IBD especially since the introduction of biologic agents. Unfortunately, despite current advances loss of response to biologics still affects up to $30-40 \%$ of patients in clinical practise [5]. Identifying factors that predict the efficacy of biologics is important to efficiently use these agents along with minimising side-effects and improving cost-effectiveness. To date certain disease related factors which impact response to anti-TNF agents include gender, age at diagnosis or the presence of fistulating disease $[5,6]$ but overall data is limited. Interestingly, despite the anatomical, genetic, and immunological differences between $\mathrm{CD}$ and UC there is no evidence to date indicating biologics work more effectively in either sub-type of IBD. Our study is the first to investigate the impact of IBD subtype on sustainability of biologics and our findings show that in both biologic-naïve and biologic-experienced patients' biologic therapy is more durable in CD than UC. In our biologic-naïve cohort patients with CD were significantly less likely to discontinue a biologic agent compared with UC and similar results were seen in patients who were biologicexperienced. More research is required to identify unique reasons why biologic therapy appears more sustainable in CD but one can hypothesis it is most likely multi-factorial. Certain disease related differences between CD and UC including genetic, immunogenic and differences in the gut microbiota can impact response to treatment. 
Other modifiable factors such as differences in the inflammatory burden between CD and UC and how each physician manages and adjusts their patient's treatment can certainly influence response to treatments. In CD, it is well known that surgery is not curative, and it is standard practice to favor bowel-sparing surgical approaches, and resection of the affected part of bowel only is preferable [7] Interestingly after the introduction of anti-TNF therapy the risk for IBD related hospitalization have only decreased in CD but not in UC [8]. Unlike CD with UC surgery is often considered curative and this can impact both physician and patient decision making and treatment goals. From recent studies there is also a clear difference in the prescribing practise of biologics between UC and CD. One cross-sectional study analysing United States (US) health care utilization in 964,633 IBD patients between 2010 and 2012 showed that UC patients were much less frequently treated with biologics than CD patients (3.5\% vs $16.8 \%$ ) [9]. A second study in the US of over 400,000 IBD patients showed that the proportion of patients using biologics increased for both UC and CD between 2007 to 2015, but to a much higher extent in patients with CD (from $21.8 \%$ to $43.8 \%$ ) than in patients with UC (from $5.1 \%$ to $16.2 \%$ ) [10]. Numerous disease related factors impact response to biologic therapy other than the sub-type of IBD. In our study shorter disease durations and older age at diagnosis had a negative impact on sustainability of biologics.

Regarding disease duration studies are conflicting. Several studies have shown in CD shorter disease duration improves response to biologics [11-13] however the opposite has been in seen in two recent studies, one looking at anti-TNF therapy and one looking at vedolizumab response $[14,15]$. In our study, like Nguyen et al's study patients with a shorter disease duration were more likely to discontinue biologic agents sooner. In our study older age at diagnosis was associated with a slightly increased risk of discontinuing biologic agents in our biologic-naïve cohort. Desai et al showed similar results in a small study looking where an age over 60 years correlated with an increased risk of discontinuation of biologics [16]. Co-therapy with immunomodulators is advised by ECCO guidelines for management of patients with moderate to severe IBD treated with IFX to help achieve and maintain remission however not for adalimumab [17]. Our study found in our biologic-naïve cohort co-therapy with immunomodulators had a negative impact on sustainability of biologics with a HR 1.6 which is expected given $48 \%$ of our biologic-naïve cohort were treated with adalimumab and $47 \%$ with IFX. In our study $37 \%$ of patients discontinued a particular biologic agent over the 5-year follow-up. The incidence of primary nonresponse to anti-TNFs varies from 10 to $30 \%$ in clinical trials and clinical practise [18-20].

The annual risk of secondary loss of response in one particular review for IFX was $13 \%$ per patient year [21] and $20.3 \%$ for adalimumab in two separate study [22]. This data highlights the need for a personalised approach to treatment for each patient with IBD. Promising targets for new therapeutic strategies are currently being developed and our study highlights the need to broaden treatment options available for IBD to ensure patients both achieve and sustain mucosal healing given the chronic nature of IBD. Limitations of our study include the retrospective and observational nature without randomization or blinding. Secondly data was not collected on disease severity at onset of biologics which can impact response to treatment. Another limitation is the variability in prescribing biologics and escalation of treatment between different gastroenterologists at our centre.

\section{Conclusion}

In conclusion our real word data indicates sustainability of biologic therapies is less in UC than CD and this is not strongly determined by prior biologic exposure. The reason biologics are more sustainable in $\mathrm{CD}$ is most likely multifactorial however this finding is important in determining how biologic agents are employed in both IBD subtypes. Our data also highlights the need for new biologics and small molecules inhibitors as there is currently an unmet medical need for treatments in UC and CD given the high rates of primary and secondary loss of response.

\section{Conflicts of Interest}

The authors have no conflicts to disclose.

\section{Funding}

The authors have no funding related to this work to disclose.

\section{References}

1. GBD 2017 Inflammatory Bowel Disease Collaborators (2020) The global, regional, and national burden of inflammatory bowel disease in 195 countries and territories, 1990-2017: A systematic analysis for the Global Burden of Disease Study 2017. Lancet 5(1): 17-30.

2. Rutgeerts P, Vermeire S, Van Assche G (2007) Mucosal healing in inflammatory bowel disease: Impossible ideal or therapeutic target? Gut 56: 453-455.

3. Brady J, Stott Miller M, Mu G, Perera S (2018) Treatment Patterns and Sequencing in Patients with Inflammatory Bowel Disease. Clinical Therapeutics 40: 1509-1521.

4. Chen C, Hartzema AG, Xiao H, Wei YJ, Chaudhry N, et al. (2019) Real-world Pattern of Biologic Use in Patients With Inflammatory Bowel Disease: Treatment Persistence, Switching, and Importance of Concurrent Immunosuppressive Therapy. Inflamm Bowel Dis 25(8): 1417-1427.

5. Lopetuso LR, Gerardi V, Papa V, Scaldaferri F, Rapaccini GL, et al. (2017) Can We Predict the Efficacy of Anti-TNF- $\alpha$ Agents? Int J Mol Sci 18(9): 1973.

6. Beaugerie L, Sokol H (2012) Clinical, serological, and genetic predictors of inflammatory bowel disease course. World J Gastroenterol 18(29): 3806-3813.

7. Gionchetti P, Dignass A, Danese S, Fernando José Magro Dias, Gerhard Rogler, et al. (2017) European evidence-based consensus on the diagnosis and management of Crohn's disease 2016: Part 2: Surgical management and special situations. J Crohns Colitis 11: 135-149. 
8. Mandel MD, Balint A, Golovics PA, Vegh Z, Mohas A, et al. (2014) Decreasing trends in hospitalizations during anti-TNF therapy are associated with time to anti-TNF therapy: Results from two referral centres. Dig Liver Dis 46(11): 985-990.

9. Van Deen WK, Van Oijen MGH, Myers KD, Adriana Centeno, William Howard, et al. (2014) A nationwide 2010-2012 analysis of U.S. health care utilization in inflammatory bowel diseases. Inflamm Bowel Dis 20(10): 1747-1753.

10. Yu H, MacIsaac D, Wong JJ, ZM Sellers, AA Wren, et al. (2018) Market share and costs of biologic therapies for inflammatory bowel disease in the USA. Aliment Pharmacol Ther 47(3): 364-370.

11. Schreiber S, Colombel JF, Bloomfield R, Nikolaus S, Schölmerich J, et al. (2010) Increased response and remission rates in short-duration Crohn's disease with subcutaneous certolizumab pegol: An analysis of PRECiSE 2 randomized maintenance trial data. Am J Gastroenterol 105(7): 1574-1582.

12. Colombel JF, Sandborn WJ, Rutgeerts P, Enns R, Hanauer SB, et al. (2007) Adalimumab for maintenance of clinical response and remission in patients with Crohn's disease: the CHARM trial. Gastroenterology 132(1): 52-65.

13. Papamichael K, Vande Casteele N, Gils A, Tops S, Hauenstein S, et al. (2015) Long-term outcome of patients with Crohn's disease who discontinued infliximab therapy upon clinical remission. Clin Gastroenterol Hepatol 13(6): 1103-1110.

14. Nguyen NH, Kurnool S, Dulai PS, Boland BS, Sandborn WJ, et al. (2020) Short Disease Duration Is Associated with Increased Risk of Treatment Failure in Biologic-Treated Patients with Ulcerative Colitis. Inflamm Bowel Dis 26(9): 1429-1435.

ISSN: 2574-1241

DOI: 10.26717/BJSTR.2021.37.006071

Jayne Doherty. Biomed J Sci \& Tech Res

CC (P) This work is licensed under Creative Commons Attribution 4.0 License

Submission Link: https://biomedres.us/submit-manuscript.php
15. Faleck DM, Winters A, Chablaney S, Shashi P, Meserve J, et al. (2019) Shorter Disease Duration Is Associated With Higher Rates of Response to Vedolizumab in Patients With Crohn's Disease But Not Ulcerative Colitis. Clin Gastroenterol Hepatol 17(12): 2497-2505.e1.

16. Desai A, Zator ZA, De Silva P, Nguyen DD, Korzenik J, et al. (2013) Older age is associated with higher rate of discontinuation of anti-TNF therapy in patients with inflammatory bowel disease. Inflamm Bowel Dis 19(2): 309-315.

17. Harbord M, Eliakim R, Bettenworth D, Karmiris K, Katsanos K, et al. (2017) Third European Evidence-based Consensus on Diagnosis and Management of Ulcerative Colitis. Part 2: Current Management. J Crohns Colitis 11(7): 769-784.

18. Sprakes MB, Ford AC, Warren L, Greer D, Hamlin J (2012) Efficacy, tolerability, and predictors of response to infliximab therapy for Crohn's disease: A large single centre experience. J Crohns Colitis 6(2): 143-53.

19. Hanauer SB, Feagan BG, Lichtenstein GR, Mayer LF, Schreiber S, et al. (2002) Maintenance infliximab for Crohn's disease: The ACCENT I randomised trial. Lancet 359(9317): 1541-1549.

20. Ford AC, Sandborn WJ, Khan KJ, Hanauer SB, Talley NJ, et al. (2011) Efficacy of biological therapies in inflammatory bowel disease: Systematic review and meta-analysis. Am J Gastroenterol 106(4): 644659.

21. Jakobovits SL, Jewell DP, Travis SP (2007) Infliximab for the treatment of ulcerative colitis: Outcomes in Oxford from 2000 to 2006. Aliment Pharmacol Ther 25(9): 1055-1060.

22. Billioud V, Sandborn WJ, Peyrin Biroulet L (2011) Loss of response and need for adalimumab dose intensification in Crohn's disease: A systematic review. Am J Gastroenterol 106(4): 674-684.

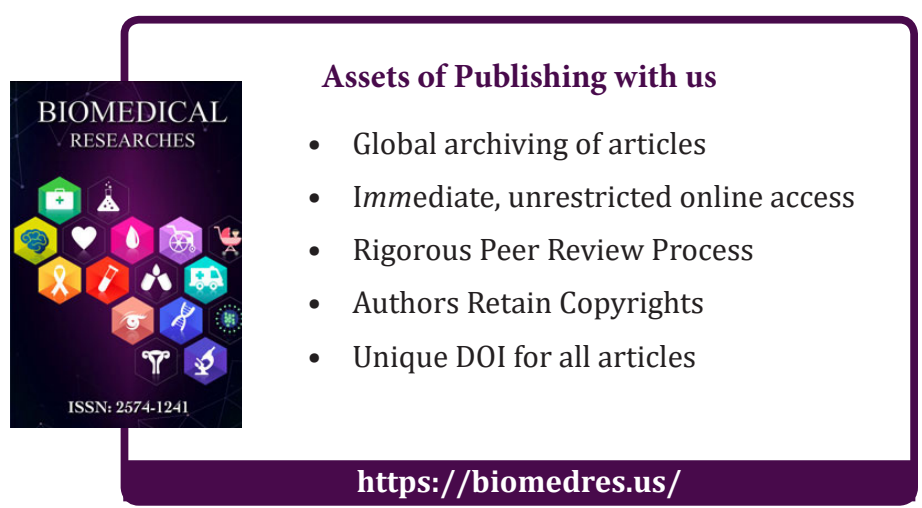

